\title{
Integrated Cryptococcal Antigen Screening and Pre-Emptive Treatment Prior to Initiation of Antiretroviral Treatment in Cambodia
}

\author{
Bopha Chim ${ }^{1 *}$, Soeung Sun Piseth ${ }^{1}$, Veng Chhun Heng ${ }^{1}$, Thai Sopheak ${ }^{1}$, Lutgarde Lynen ${ }^{2}$ and Johan van Griensven ${ }^{1,2^{*}}$ \\ ${ }^{1}$ Department of Infectious Diseases, Sihanouk Hospital Center of HOPE, Phnom Penh, Cambodia \\ ${ }^{2}$ Department of Clinical Sciences, Institute of Tropical Medicine, Antwerp, Belgium
}

\begin{abstract}
Background: Detection of serum cryptococcal antigen (sCrAg) in HIV-infected patients is associated with 3-6 fold increased mortality. Studies on the feasibility and effectiveness of sCrAg-screening and pre-emptive therapy before antiretroviral treatment (ART) are limited. Amongst antiretroviral treatment-naïve adults with baseline CD4 counts $<100$ cells/ $\mu \mathrm{L}$ undergoing pre-ART sCrAg screening, without previous cryptococcal meningitis (CM), we report on the 1) prevalence of sCrAg-positivity; 2) proportion with concurrent $\mathrm{CM}$; 3) six-month patient outcomes.

Methods: This is a retrospective hospital-based cohort study evaluating a "screen-and-treat" protocol implemented between 10/2008 and 8/2012 in Phnom Penh, Cambodia. All sCrAg(+) patients underwent lumbar puncture (LP). After ruling-out $\mathrm{CM}$, fluconazole was started $(400 \mathrm{mg} / \mathrm{d}-10$ weeks; $200 \mathrm{mg} / \mathrm{d}$ secondary prophylaxis) immediately. $\mathrm{sCrAg}(-)$ patients received primary prophylaxis (fluconazole $100 \mathrm{mg} / \mathrm{d})$. CM was treated with amphotericin-B $(0.7 \mathrm{mg} /$ $\mathrm{kg}-2$ weeks, followed by fluconazole $400 \mathrm{mg} /$ day for 8 weeks).

Results: A total of 357 patients were enrolled with a median age of 38 (IQR 31-43) years and median CD4 count of 28 (IQR $14-53$ ) cells $/ \mu \mathrm{L} ; 54 \%$ were male, $20 \%$ had BMI $<16 \mathrm{~kg} / \mathrm{m}^{2}$. Thirty $(8.4 \%)$ of 357 tested were $\mathrm{sCrAg}(+)$. Six $(20 \%)$ of the $30 \mathrm{sCrAg}(+)$ patients had concurrent $\mathrm{CM}$ with no/minimal symptoms. Two were treated as $\mathrm{CM}$ without LP done. Amongst SCrAg(+) patients, the following 6-month outcomes were noted: $25(83.3 \%)$ were retained and on ART, $1(3.3 \%)$ patient died and $4(13.3 \%)$ were lost-to-follow-up. Excluding those treated for $\mathrm{CM}(\mathrm{n}=8)$, one case $(1 / 22 ; 4.5 \%)$ of incident CM was detected by month six. Corresponding 6 -month outcomes amongst the $327 \mathrm{CrAg}(-)$ patients were: $269(82.3 \%)$ retained and on ART, 23 (7\%) deaths, 29 (9\%) lost-to-follow-up, 6 (1.8\%) transferred-out. One case $(1 / 327 ; 0.3 \%)$ of incident CM was documented
\end{abstract}

Conclusion: Outcomes of $\mathrm{sCrAg}(+)$ patients with the "screen-and-treat" approach were good. Fluconazole at $400 \mathrm{mg} /$ day appears appropriate for treatment of $\mathrm{sCrAg}(+)$ patients. Routine $\mathrm{LP}$ in sCrAg(+) might be indicated.

Keywords: Cryptococcal infection; Screening; Algorithm; Cryptococcal meningitis

\section{Introduction}

Cryptococcal disease is one of the most important opportunistic infections in HIV-infected individuals, especially in sub-Saharan Africa and certain parts of Asia. It has been estimated to account for between $13 \%$ and $44 \%$ of deaths in HIV-infected patients in resourcelimited countries [1]. The most common presentation is cryptococcal meningitis (CM), with a case fatality rate between $35 \%-65 \%$ in such settings [2-4].

Increasingly, a "screen-and-treat" strategy has been advocated in CM-prevalent areas, based on the following evidence [5,6]. First, cryptococcal antigenemia can be reliably detected, and prototype lateral flow assays that can be used on blood and urine have been developed $[7,8]$. Second, detection of cryptococcal antigen in the serum ( $\mathrm{sCrAg}$ ) is clinically relevant and precedes the onset of overt disease [9]. In otherwise asymptomatic HIV-infected persons, cryptococcal antigenemia independently increased mortality 4 to 7 fold [10-12]. Untreated, up to $30 \%$ developed CM over the next few weeks to months, even when initiating antiretroviral treatment (ART) [11]. Importantly, there are some indications that pre-emptive treatment of asymptomatic cryptococcal antigenemia with fluconazole - widely available in HIV care programs - can prevent clinical progression towards $\mathrm{CM}$ and reduce mortality [13-16].

The World Health Organization (WHO) recently issued a rapid advice recommending targeted screening for cryptococcal antigenemia in ART-naïve patients with a CD4 count less than 100 cells/ $\mu \mathrm{L}$ before
ART initiation, followed by pre-emptive anti-fungal therapy if sCrAgpositive [17]. However, given the limited evidence-base, this came as a conditional recommendation. WHO also stated that "further field studies are needed to establish: (i) the feasibility of this "screen-and-treat" approach and its impact on mortality; (ii) the optimal selection of serum CrAg-positive patients who require a lumbar puncture to rule-out central nervous system disease; and (iii) the optimal treatment regimen in those with isolated serum CrAg positivity" [17].

Cambodia is one of the East-Asian countries with a high burden of CM in HIV-infected patients [18]. A standardized protocol for sCrAg screening and treatment was implemented in October 2008 in a large urban HIV treatment program in Phnom Penh, Cambodia. sCrAg(+) patients underwent lumbar puncture (LP), isolated $\mathrm{sCrAg}$ was treated

*Corresponding authors: Johan van Griensven, Department of Clinical Sciences, Institute of Tropical Medicine, 2000 Antwerp, Belgium, Tel: +855 11 345728; Fax: +855 23 882485; E-mail: jvangriensven@itg.be

Bopha Chim, Department of Infectious Diseases, Sihanouk Hospital Center of HOPE Phnom Penh, P.O.Box: 2318, St 134, Vealvong, 7 Makara, Phnom Penh, Cambodia, Tel: 855-12-953905; Fax: 855-23-882485; E-mail: bophachim@sihosp.org

Received June 17, 2013; Accepted July 24, 2013; Published July 27, 2013

Citation: Chim B, Piseth SS, Heng VC, Sopheak T, Lynen L, et al. (2013) Integrated Cryptococcal Antigen Screening and Pre-Emptive Treatment Prior to Initiation of Antiretroviral Treatment in Cambodia. J AIDS Clin Res 4: 227. doi: 10.4172/21556113.1000227

Copyright: ( ) 2013 Chim B, et al. This is an open-access article distributed unde the terms of the Creative Commons Attribution License, which permits unrestricted use, distribution, and reproduction in any medium, provided the original author and source are credited. 
with fluconazole. The objective of this study was to report on the feasibility and effectiveness of this strategy. Amongst adult ART-naïve individuals with a baseline CD4 count below 100 cells/ $\mu \mathrm{L}$ undergoing pre-ART sCrAg-screening, we report on the 1) prevalence and risk factors for sCrAg-positivity; 2) proportion with concurrent CM; 3) sixmonth patient outcomes.

\section{Methods}

\section{Study design and population}

We conducted a retrospective cohort study at the SihanoukHospital-Center-of-Hope in Phnom Penh, Cambodia. Since March 2003, this non-governmental hospital provides free comprehensive HIV care as part of the national ART program. All ART-naive adults (in patients or outpatients) with a baseline CD4 count below 100 cells $/ \mu \mathrm{L}$ that were screened for $\mathrm{sCrAg}$ before ART initiation between October 2008 and August 2012 were included. Those with a history of previous $\mathrm{CM}$ or invasive cryptococcal infection prior to testing, or that were taking fluconazole treatment at the time of enrolment were excluded.

\section{Algorithm for screening and treatment of cryptococcal antigenemia}

All newly enrolled HIV-positive patients were clinically staged upon enrolment, evaluated for ongoing opportunistic infections, and had a CD4 cell count done (FACSCount, Becton Dickinson, Franklin Lakes, NJ). Patients with a CD4 count below 100 cells $/ \mu \mathrm{L}$ were started on primary prophylaxis with fluconazole (100 $\mathrm{mg}$ /day), in line with the national guidelines. As part of the pre-ART work-up, three adherence counseling sessions were planned. During the second session, all individuals underwent baseline pre-ART laboratory investigations, including hematology, liver and renal function tests. Those with a baseline CD4 count below 100 cells/ $\mu \mathrm{L}$ were additionally screened for sCrAg using Pastorex Crypto Plus (Bio-Rad Laboratories, Hercules, CA, USA). This test was reported as positive or negative, no titration was done. As per national guideline, those with a negative screening test continued primary prophylaxis with fluconazole until the CD4 count was above 100 cells/ $\mu \mathrm{L}$ on two occasions measured at six-month intervals.

Patients with a positive screening test systematically underwent LP, irrespective of symptoms suggestive of CM (Figure 1). Examinations conducted on the cerebrospinal fluid consisted of: white cell count and differentiation, protein and glucose concentration, cryptococcal antigen, India ink direct examination, and culture. Patients diagnosed with $\mathrm{CM}$ received amphotericin $\mathrm{B}(0.7 \mathrm{mgkg} /$ day for two weeks), followed by fluconazole ( $400 \mathrm{mg}$ /day for eight weeks). If CM was ruledout, treatment consisted of fluconazole $400 \mathrm{mg} /$ day for ten weeks, started on the same day as the LP. This was followed by fluconazole 200 $\mathrm{mg} /$ day until a CD4 count above 100 cells/ $\mu \mathrm{L}$ was documented on at least two occasions at six-month intervals.

All patients systematically underwent a chest X-ray and additional laboratory investigations (sputum evaluation) to rule out concurrent infections, particularly tuberculosis. Additional investigations for extraneural cryptococcal infection were done as clinically indicated (eg skin biopsy). Cultures of urine or respiratory samples to detect cryptococcal infection were not systematically done.

All physicians were involved in implementing the protocol, which was used both for outpatients as well as hospitalized patients. There was no fluconazole dose adjustment done for those with concurrent tuberculosis.

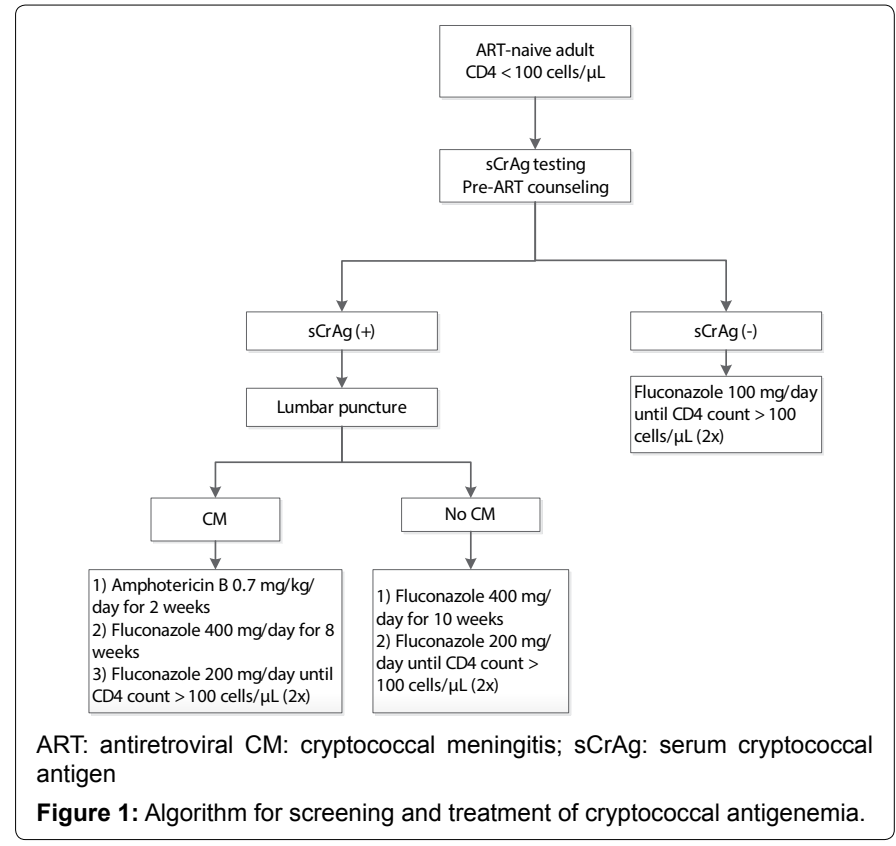

\section{Antiretroviral treatment initiation and monitoring}

Treatment initiation of ART followed WHO recommendations $[19,20]$. For CM, ART was initiated after initial clinical improvement, typically at least two weeks after starting CM treatment. For isolated sCrAg, ART was started early after fluconazole initiation (typically within one to two weeks). First line treatment consisted of a generic fixed-dose combination containing stavudine, lamivudine and nevirapine. For tuberculosis cases, nevirapine was temporarily replaced by efavirenz. Patients were seen monthly during the first three months after ART initiation. Subsequently, visits were scheduled every 2-3 months for clinically stable patients. A number of issues were systematically addressed, including screening for opportunistic infections, treatment toxicity and adherence. All care was provided by physicians. A full blood count and CD4 cell count was done every six months after ART initiation, with liver function tests performed more regularly. All patients with WHO stage II/III/IV and all those with a CD4 count $<200$ cells $\mu \mathrm{L}$ were given cotrimoxazole prophylaxis. From March 2011, isoniazid prophylactic therapy was prescribed for individuals in pre-ART care, if tuberculosis was ruled-out. Patients not presenting at their scheduled visits were traced by home visits or by phone, and considered lost to follow-up (LTFU) if they were not found within six months after their most recent visit. Additional details of the ART program at SHCH have been published before [21-23].

\section{Outcomes and operational definitions}

$\mathrm{CM}$ was defined as a positive result on cerebrospinal fluid of either India Ink staining, cryptococcal antigen testing or culture. If CM was detected at the same time as the positive sCrAg screening test, this was labeled as prevalent CM. Cases of CM detected during follow-up, after the initial screening, were defined as incident CM. $\mathrm{sCrAg}(+)$ individuals with $\mathrm{CM}$ ruled-out and no other associated organ involvement were defined as isolated $\operatorname{sCrAg}(+)$.

\section{Data collection and statistical analysis}

At the ART program onset, structured clinical records, data collection tools, and a database were developed. On a daily basis, clinical, laboratory and treatment data were collected and electronically stored. 
Citation: Chim B, Piseth SS, Heng VC, Sopheak T , Lynen L, et al. (2013) Integrated Cryptococcal Antigen Screening and Pre-Emptive Treatment Prior to Initiation of Antiretroviral Treatment in Cambodia. J AIDS Clin Res 4: 227. doi: 10.4172/2155-6113.1000227

Page 3 of 6

Physicians were trained in standardized patient assessment using the hospital guidelines and protocols. Quality control of the stored data was performed at regular intervals. For this study, data were extracted from this database. In addition, HIV clinical records were reviewed for all $\mathrm{sCrAg}(+)$ individuals to retrieve additional data relating to symptoms of CM and LP procedures.

Analysis focused on the following outcomes: prevalence of and risk factors for cryptococcal antigenemia; patient characteristics and proportion with prevalent CM; patient outcomes by six months after sCrAg testing of those with and without cryptococcal infection, defined as retained and on ART, lost to care (death or LTFU) and transferred-out. The frequency of incident CM by six months after sCrAg-testing was calculated as well. For all $s \mathrm{CrAg}(+)$ patients, compliance with the algorithm was assessed in terms of diagnostic work-up and treatment prescribed. Medians (interquartile range (IQR)) and frequencies (\%) were used to describe patients' characteristics. Baseline patient characteristics were compared using Chi square or Fisher's exact tests for categorical variables and Wilcoxon rank-sum test for continuous variables. Kaplan-Meier methods were used to assess the patient outcomes over time, starting from the time of sCrAg testing.

\section{Ethical issues}

Since the launching of the HIV care program, clinical data have been routinely collected for purposes of program monitoring and evaluation, and research activities. Patients were requested to give informed consent to store and use the data. Data collection and informed consent procedures were approved by the Institutional Review Board of the $\mathrm{SHCH}$ and the Institute of Tropical Medicine, Antwerp, Belgium. No patient identifiers were included in the dataset used for this analysis.

\section{Results}

Between October 2008 and August 2012, a total of 357 individuals with a baseline CD4 count $<100$ cells/ $\mu \mathrm{L}$ underwent screening for sCrAg as part of the pre-ART preparatory work-up. None were clinically suspected of $\mathrm{CM}$ at the time of testing. The median $\mathrm{CD} 4$ count at the time was 28 cells $/ \mu \mathrm{L}$. A body mass index $(\mathrm{BMI})<16 \mathrm{~kg} / \mathrm{m}^{2}$ was seen in $20.2 \%(72 / 357)$, see Table 1 .

\section{Prevalence and risk factors for cryptococcal infection during screening}

In total, $30(8.3 \%)$ had a positive screening result. Factors associated with sCrAg-positivity included a BMI $<16 \mathrm{~kg} / \mathrm{m}^{2}$ and a CD4 count $<$

\begin{tabular}{|c|c|c|c|c|}
\hline & $\begin{array}{c}\text { Total } \\
(\mathrm{n}=357 ; \mathrm{n}(\%))\end{array}$ & $\begin{array}{l}\text { Cryptococcal antigen }(+) \\
(n=30 ; n(\%))\end{array}$ & $\begin{array}{c}\text { Cryptococcal antigen (-) } \\
(n=327 ; n(\%))\end{array}$ & $P$-value \\
\hline \multicolumn{5}{|l|}{ Sex } \\
\hline Female & $164(46)$ & $17(56.7)$ & $147(45.0)$ & 0.218 \\
\hline Male & $193(54)$ & $13(43.3)$ & $180(55.0)$ & \\
\hline Age (years); median (IQR) & $38(31-43)$ & $38(31-41)$ & $38(31-43)$ & 0.896 \\
\hline CD4 count (cells $/ \mu \mathrm{L})$; median (IQR) & $28(14-53)$ & $15(7-23)$ & $32(14-58)$ & $<0.001$ \\
\hline$<50$ cells $/ \mu \mathrm{L}$ & $257(72)$ & $28(93.3)$ & $231(70.6)$ & $<0.01$ \\
\hline $\mathrm{BMI} \mathrm{kg} / \mathrm{m}^{2} ;$ median (IQR) & $18.3(16.6-20.1)$ & $16.7(14.7-18.5)$ & $18.4(16.7-20.3)$ & $<0.001$ \\
\hline$<16 \mathrm{~kg} / \mathrm{m}^{2}$ & $72(20.2)$ & $12(40.0)$ & $60(18.6)$ & $<0.01$ \\
\hline Started on ART & $337(94.4)$ & $28(93.3)$ & $309(94.5)$ & 0.791 \\
\hline ART regimen $(n=337)$ & & & & 0.808 \\
\hline D4T/3TC/NVP & $146(43.3)$ & $11(39.3)$ & $135(43.7)$ & \\
\hline D4T/3TC/EFV & $174(51.6)$ & $16(57.1)$ & $158(51.1)$ & \\
\hline Other & $17(5.0)$ & $1(3.6)$ & $16(5.2)$ & \\
\hline
\end{tabular}

ART: Antiretroviral Treatment; BMI: Body Mass Index; CM: Cryptococcal Meningitis; D4T: Stavudine; EFV: Efavirenz; IQR: Interquartile Range; NVP: Nevirapine; 3TC: Lamivudine

Table 1: Baseline characteristics of individuals screened for serum cryptococcal antigen (sCrAg) prior to ART initiation with a CD4 count $<100$ cells/ $\mu \mathrm{L}$, Phnom Penh, 2008-2012 ( $\mathrm{N}=357)$.

Deviations in treatment (fluconazole $400 \mathrm{mg} / \mathrm{d} 10$ weeks)

\begin{tabular}{|l|l} 
Amphotericin B without evidence of CM (no LP done) & $2(6.7)$
\end{tabular}

\begin{tabular}{|l|l}
\hline Fluconazole $800 \mathrm{mg}$ for $3 \mathrm{~d}$ followed by $400 \mathrm{mg} / \mathrm{d}$ & $5(16.7)$
\end{tabular}

Failure to complete 10 weeks of fluconazole $400 \mathrm{mg} / \mathrm{d}$

\begin{tabular}{|l|l}
\hline Discontinued by physician prematurely (at 6.5 weeks) & 1 (3.3)
\end{tabular}

\begin{tabular}{|l|l}
\hline Livertoxicity (at 5 weeks) & 1 (3.3)
\end{tabular}

Concurrent potentially hepatotoxic medication:

co-trimoxazole, nevirapine

Failure to continue secondary prophylaxis until CD4 recovery

\begin{tabular}{l|l}
\hline Livertoxicity & $3(10.0)$
\end{tabular}

Concurrent potentially hepatotoxic medication:

co-trimoxazole $(n-3)$, isoniazid $(n-1)$, nevirapine $(n-1)$, efavirenz $(n-2)$

CM: Cryptococcal Meningitis; Lumbar Puncture (LP)

${ }^{a}$ All data: $n(\%)$

Table 2: Compliance with and feasibility of the implementation of the "screen-and-treat" guidelines $(\mathrm{N}=30)^{\mathrm{a}}$ 
50 cells/ $\mu \mathrm{L}$. Concurrent ("prevalent”) CM was diagnosed in $6(20 \%)$ of the 30 individuals that were $\mathrm{sCrAg}(+)$. Of interest, two of the six $\mathrm{CM}$ cases were asymptomatic, whereas four had only minor symptoms (mild headache, feeling feverish) that could possibly hint to CM. In addition, two asymptomatic patients were treated as CM but had no LP done (one for unclear reasons, one patient refused), Table 2. ART was initiated at a median of 19 days after starting treatment for CM.

\section{Incident cryptococcal infection and patient outcomes by six months after screening}

Amongst the $30 \mathrm{sCrAg-positives,} \mathrm{the} \mathrm{following} \mathrm{6-months} \mathrm{outcomes}$ were documented: 25 (83.3\%) were retained and on ART; one $(3.3 \%)$ patient died and four (13.3\%) were LTFU (Figures 2 and 3). The single death occurred 32 days after testing sCrAg-positive (but with CM ruled-out). The patient died of presumed disseminated tuberculosis and sepsis, 16 days after ART initiation. Loss to follow-up occurred at respectively $32,36,80$ and 98 days after sCrAg-testing. All but one were in good health at the latest clinical encounter.

Twenty-eight (93\%) of the 30 positive $\mathrm{sCrAg}(+)$ individuals initiated

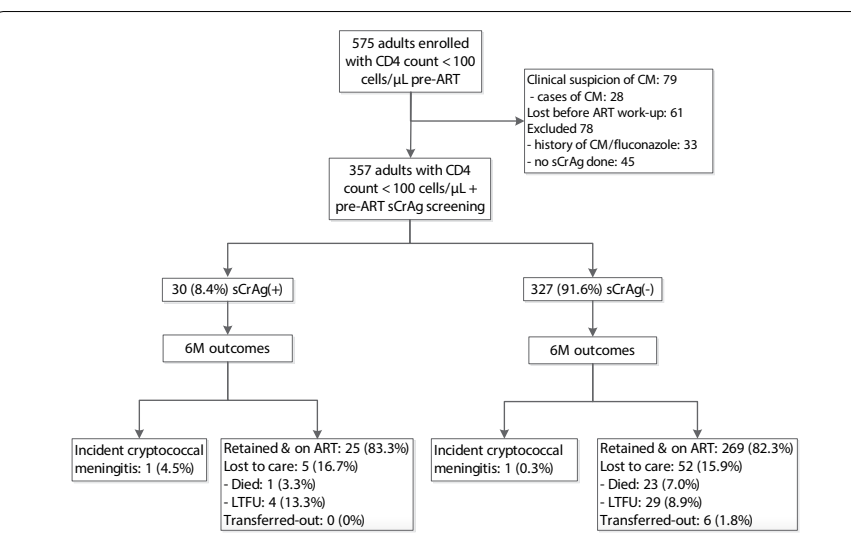

Figure 2: Flowchart representing the number of individuals screened for serum cryptococcal antigen (sCrAg) prior to ART initiation and six month patient outcomes.

ART: antiretroviral treatment; CM: cryptococcal meningitis; LTFU: lost to follow-up. ${ }^{a} \mathrm{n}=22$ (after exclusion of cases with confirmed or presumed prevalent CM)

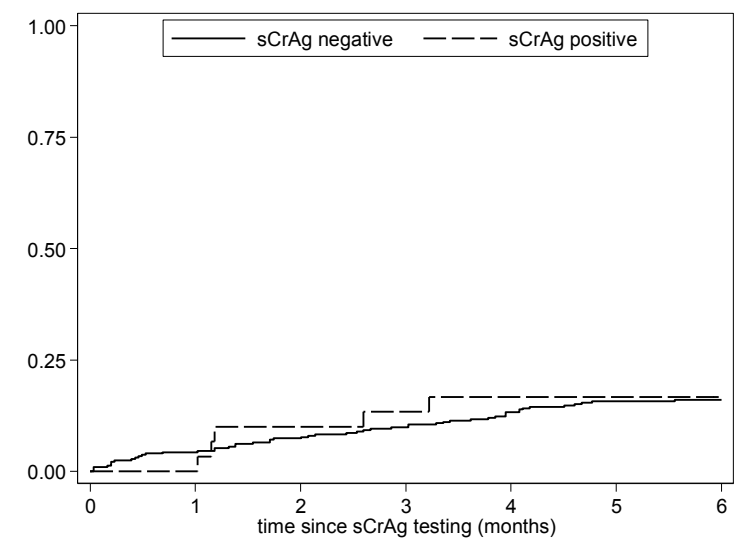

Figure 3: Kaplan-Meier estimates of cumulative incidence of death or lost to follow-up at different time points after testing for serum cryptococcal antigen (sCrAg) prior to initiation of antiretroviral treatment.
ART (two were LTFU after testing). ART was initiated a median of 25 days after sCrAg-testing. Excluding those treated for $\mathrm{CM}(\mathrm{n}=8)$, one case $(1 / 22 ; 4.5 \%)$ of incident CM was detected by month six (Figure 2 ). This patient had adhered well to fluconazole consolidation therapy and developed symptoms of CM early after starting fluconazole secondary prophylaxis $(200 \mathrm{mg} /$ day). None of the six patients with prevalent CM displayed signs of the immune reconstitution inflammatory syndrome (IRIS) of the central nervous system within the first six months after initiation of ART.

Of the $327 \mathrm{sCrAg}(-)$ patients, $269(82.3 \%)$ were retained and on ART by six months after testing (Figure 2). There were 23 (7\%) deaths and $29(9 \%)$ LTFU, $6(1.8 \%)$ patients were transferred-out. Ninety four percent $(309 / 327)$ of the $\mathrm{sCrAg}(-)$ patients started ART. ART was initiated a median of 10 days after sCrAg-testing. One case (1/327; $0.3 \%$ ) of incident $\mathrm{CM}$ was identified in the $\mathrm{sCrAg}(-)$ group, in a patient with reportedly good adherence to fluconazole prophylaxis.

\section{Feasibility of and compliance with the implementation of the "screen-and-treat" guidelines}

Although overall the protocol was correctly implemented for the majority of patients, a number of deviations were observed (Table 2). Five $\mathrm{sCrAg}(+)$ individuals without $\mathrm{CM}$ received fluconazole $800 \mathrm{mg}$ for three days, two patients had no LP done and were treated with amphotericin B. Secondary prophylaxis was generally continued appropriately, although some had to discontinue due to liver toxicity (see below). In general, it was given for a median time of 340 (IQR 238514) days.

Liver toxicity requiring discontinuation of fluconazole occurred in four $(13.3 \%)$ individuals, but concurrent use of potentially hepatotoxic drugs was common. To note that besides ART, the use of potentially hepatotoxic medication together with fluconazole was common among the 30 cases: all initiated cotrimoxazole, four were given isoniazid prophylactic therapy and 18 received antituberculous treatment.

\section{Discussion}

This study reports on the implementation of a "screen-andtreat" approach for cryptococcal infection in a Cambodian hospital, as currently recommended by the WHO. In ART-naïve adults with CD4 counts $<100$ cells being prepared for ART initiation, screening for sCrAg identified 30 (8.3\%) sCrAg(+) patients in 357 screened. Treatment with fluconazole was associated with a very low incidence of $\mathrm{CM}$ over the next six months, and good survival rates were observed. Overall, the protocol was fairly well implemented, fluconazole was discontinued due to liver toxicity in $4(13.3 \%) \mathrm{sCrAg}(+)$ patients. The findings support the feasibility and effectiveness of such a strategy in reducing the incidence of $\mathrm{CM}$ and probably reducing the associated mortality.

The prevalence of $8.4 \%$ amongst otherwise asymptomatic patients is similar to studies in patients with CD4 cell counts $<100$ cells $/ \mu \mathrm{L}$ in rural Uganda (5.3\%), South-Africa (7\%) and Thailand (9.2\%) $[10,11,13]$. Other studies, including both symptomatic and asymptomatic patients, often directly at enrolment, have found higher estimates amongst those with CD 4 cell counts $<100$ cells $/ \mu \mathrm{L}$, up to $18.8 \%$ and $20.6 \%$ in Uganda and Cambodia respectively $[14,24]$. In the latter study, the prevalence of $\operatorname{sCrAg}(+)$ in those without clinical suspicion of $\mathrm{CM}$ was $10.8 \%$, comparable to our findings [14].

Although we acknowledge that the lack of a comparison treatment group for the sCrAg-positive patients warrants cautious interpretation 
of the treatment outcome data, our findings remain encouraging. Asymptomatic sCrAg has consistenty been identified as an independent risk factor for mortality, with a 3 to 7 -fold increase in risk $[11,13]$. Availably evidence also indicates that around $30 \%$ of $\operatorname{sCrAg}(+)$ individuals initiated on ART, but without fluconazole treatment, experience progressive cryptococcal infection leading to CM $[11,13]$. The mortality and attrition in our study was low. Indirectly, this would suggest that pre-emptive treatment with fluconazole could counter the additional mortality related to cryptococcal infection. Combined with the very low incidence of incident $\mathrm{CM}$, this suggests that fluconazole treatment at a dose of $400 \mathrm{mg}$ might be sufficient for treatment of asymptomatic sCrAg-positivity, at least in our setting. We note however that $\mathrm{CM}$ was systematically ruled-out, and treated with amphotericin $\mathrm{B}$ if diagnosed. We also acknowledge that some patients received more "aggressive" treatment, mostly by providing a loading dose of $800 \mathrm{mg} /$ day for three days. Consequently, our findings remain to be confirmed by other studies and in different settings.

In a Ugandan study, efficacy of fluconazole was observed with fluconazole (200-400 mg) given for only 2-4 weeks, although treatment was not well-standardized [15]. However, $14.3 \%(3 / 21)$ treated with fluconazole developed incident $\mathrm{CM}$, and mortality was still relatively high (29\%). Moreover, CM was not systematically ruled-out in $\mathrm{SCrAg}(+)$ patients. Much higher doses $(1200 \mathrm{mg} /$ day induction followed by 800 $\mathrm{mg}$ /day for 8 weeks) were used in a recent Kenyan study, whereby $11 \%$ of newly enrolled patients in a number of clinics with CD4 counts < 100 cells/ $\mu \mathrm{L}$ screened $\mathrm{sCrAg}(+)$ [25]. In contrast with our study, a high mortality rate $(25 \%)$ was found. There was also no survival benefit associated with the implementation of the "screen-and-treat strategy", relative to a historical control group. Although low rates of initiation of ART and fluconazole might be partly to blame, no survival benefit was seen in sensitivity analysis excluding those that failed to initiate fluconazole. Besides a number of additional factors, undiagnosed $\mathrm{CM}$ might have contributed to this. Moreover, they tested all patients immediately after enrolment, many of them being symptomatic. This obviously differs from our approach, whereby screening was done as part of the pre-ART work-up.

Our findings of asymptomatic or oligo-symptomatic prevalent $\mathrm{CM}$ in at least $20 \%$ of the $\mathrm{sCrAg}(+)$ individuals is also of interest and in keeping with previous reports [13]. On the one hand, this would argue for a low threshold for LP - perhaps even routinely - in $\mathrm{sCrAg}(+)$ patients. On the other hand, it is not known whether such asymptomatic - probably early - stages of CM can be effectively treated with fluconazole at $400 \mathrm{mg} / \mathrm{day}$ (and hence there might not be a clear benefit associated with the LP), or whether this requires amphotericin $\mathrm{B}$ or higher fluconazole doses. We note that fluconazole dosed at 400 $\mathrm{mg} /$ day is considered fungistatic only and higher doses are required for fungicidal effects. The optimal timing of ART initiation for such patients is also not defined.

Overall, the protocol was correctly implemented for most patients. The most common deviation was the prescription of a fluconazole loading dose. We assume some physicians in the program preferred this option based on some evidence that higher fluconazole dosing is required to achieve fungicidal effects $[26,27]$. Current WHO guidelines also recommend higher fluconazole dosing for the first two weeks [17]. Liver toxicity requiring discontinuation of fluconazole was not uncommon. At the time of occurrence, most patients were taking three or four potentially hepatotoxic drugs. Although use of fluconazole at $800 \mathrm{mg} /$ day for two weeks - as recommended by WHO - was found to be safe in clinical trials, [28] this remains to be confirmed in routine clinical settings. Moreover, concurrent tuberculosis was common. There are indications that, due to drug interactions, those on TB treatment might require higher dosing of fluconazole [5]. Further research on this issue is needed.

The strengths of this study include the fact that it was conducted in a routine clinical setting, and hence the findings are more likely to represent the reality on the ground. Moreover, the guideline was implemented fairly consistently and with a high uptake, demonstrating its potential impact on CM and mortality if such conditions can be ensured. There are a number of important limitations to acknowledge. As a retrospective operational study using routinely collected patient information, some data were incomplete. Although rates of LTFU compared rather favorably with many HIV cohort reports, unascertained death or CM remains a concern. Detailed reports of the causes of death might have been of interest. Moreover, the single-center design in a relatively well-resourced hospital limits the generalizability of the findings. Finally, the use of primary prophylaxis for $\mathrm{sCrAg}(-)$ patients, as recommended by the national guidelines, differs from current WHO recommendations.

In conclusion, our data demonstrate that implementation of a pre-ART "screen-and-treat strategy" for cryptococcal infection is feasible and effective in a hospital setting in Cambodia. Our findings suggest that fluconazole at a dose of $400 \mathrm{mg} /$ day is sufficient to treat cryptococcal antigenemia if $\mathrm{CM}$ has been ruled-out. However, our observations remain to be confirmed by other studies, especially from settings where LP is not systematically done. In the meanwhile, it might be safer to follow to the current WHO guideline recommending 800 $\mathrm{mg}$ /day for two weeks, followed by $400 \mathrm{mg} /$ day for eight weeks if there are no signs or symptoms of CM or CM has been ruled-out.

\section{Acknowledgements}

We thank all patients, the hospital management team and staff at the Sihanouk Hospital Center of HOPE (SHCH) for their support and for their contribution to the data collection for this study. JvG is supported by the Inbev-Baillet Latour Fund.

\section{References}

1. Park BJ, Wannemuehler KA, Marston BJ, Govender N, Pappas PG, et al (2009) Estimation of the current global burden of cryptococcal meningitis among persons living with HIVIAIDS. AIDS 23: 525-530.

2. Jarvis JN, Meintjes G, Harrison TS (2010) Outcomes of cryptococcal meningitis in antiretroviral naïve and experienced patients in South Africa. J Infect 60: 496-498.

3. Butler EK, Boulware DR, Bohjanen PR, Meya DB (2012) Long term 5-year survival of persons with cryptococcal meningitis or asymptomatic subclinical antigenemia in Uganda. PLoS One 7: e51291.

4. Park BJ, Shetty S, Ahlquist A, Greenbaum A, Miller JL, et al. (2011) Longterm follow-up and survival of antiretroviral-naive patients with cryptococcal meningitis in the pre-antiretroviral therapy era, Gauteng Province, South Africa. Int J STD AIDS 22: 199-203.

5. Rajasingham R, Meya DB, Boulware DR (2012) Integrating cryptococcal antigen screening and pre-emptive treatment into routine HIV care. J Acquir Immune Defic Syndr 59: e85-91.

6. Jarvis JN, Govender N, Chiller T, Park BJ, Longley N, et al. (2012) Cryptococcal antigen screening and preemptive therapy in patients initiating antiretroviral therapy in resource-limited settings: a proposed algorithm for clinical implementation. J Int Assoc Physicians AIDS Care (Chic) 11: 374-379.

7. Jarvis JN, Percival A, Bauman S, Pelfrey J, Meintjes G, et al. (2011) Evaluation of a novel point-of-care cryptococcal antigen test on serum, plasma, and urine from patients with HIV-associated cryptococcal meningitis. Clin Infect Dis 53 : 1019-1023.

8. Lindsley MD, Mekha N, Baggett HC, Surinthong $\mathrm{Y}$, Autthateinchai R, et al (2011) Evaluation of a newly developed lateral flow immunoassay for the diagnosis of cryptococcosis. Clin Infect Dis 53: 321-325. 
Citation: Chim B, Piseth SS, Heng VC, Sopheak T , Lynen L, et al. (2013) Integrated Cryptococcal Antigen Screening and Pre-Emptive Treatment Prior to Initiation of Antiretroviral Treatment in Cambodia. J AIDS Clin Res 4: 227. doi: 10.4172/2155-6113.1000227

9. French N, Gray K, Watera C, Nakiyingi J, Lugada E, et al. (2002) Cryptococcal infection in a cohort of HIV-1-infected Ugandan adults. AIDS 16: 1031-1038.

10. Liechty CA, Solberg P, Were W, Ekwaru JP, Ransom RL, et al. (2007) Asymptomatic serum cryptococcal antigenemia and early mortality during antiretroviral therapy in rural Uganda. Trop Med Int Health 12: 929-935.

11. Jarvis JN, Lawn SD, Vogt M, Bangani N, Wood R, et al. (2009) Screening for cryptococcal antigenemia in patients accessing an antiretroviral treatment program in South Africa. Clin Infect Dis 48: 856-862.

12. Worodria W, Massinga-Loembe M, Mazakpwe D, Luzinda K, Menten J, et al. (2011) Incidence and predictors of mortality and the effect of tuberculosis immune reconstitution inflammatory syndrome in a cohort of TB/HIV patients commencing antiretroviral therapy. J Acquir Immune Defic Syndr 58: 32-37.

13. Pongsai P, Atamasirikul K, Sungkanuparph S (2010) The role of serum cryptococcal antigen screening for the early diagnosis of cryptococcosis in HIVinfected patients with different ranges of CD4 cell counts. J Infect 60: 474-477.

14. Micol R, Lortholary O, Sar B, Laureillard D, Ngeth C, et al. (2007) Prevalence, determinants of positivity, and clinical utility of cryptococcal antigenemia in Cambodian HIV-infected patients. J Acquir Immune Defic Syndr 45: 555-559.

15. Meya DB, Manabe YC, Castelnuovo B, Cook BA, Elbireer AM, et al. (2010) Cost-effectiveness of serum cryptococcal antigen screening to prevent deaths among HIV-infected persons with a CD4+ cell count $<$ or $=100 \mathrm{cells} / \mathrm{microL}$ who start HIV therapy in resource-limited settings. Clin Infect Dis 51: 448-455.

16. Feldmesser M, Harris C, Reichberg S, Khan S, Casadevall A (1996) Serum cryptococcal antigen in patients with AIDS. Clin Infect Dis 23: 827-830.

17. World Health Organization (2011) Rapid advice: Diagnosis, Prevention and Management of Cryptococcal Disease in HIV-infected Adults, Adolescents and Children. World Health Organization, Geneva, Switzerland.

18. Espié E, Pinoges L, Balkan S, Chanchhaya N, Molfino L, et al. (2010) Cryptococcal meningitis in HIV-infected patients: a longitudinal study in Cambodia. Trop Med Int Health 15: 1375-1381.
19. Word Health Organization (2006) Antiretroviral therapy for HIV infection in adults and adolescents. Recommendations for a public health approach, World Health Organization, Geneva, Switserland.

20. Word Health Organization (2010) Antiretroviral therapy for HIV infection in adults and adolescents. Recommendations for a public health approach, World Health Organization, Geneva, Switserland.

21. Phan V, Thai S, Choun K, Lynen L, van Griensven J (2012) Incidence of treatment-limiting toxicity with stavudine-based antiretroviral therapy in Cambodia: a retrospective cohort study. PloS one 7: e30647.

22. van Griensven J, Thai S (2011) Predictors of immune recovery and the association with late mortality while on antiretroviral treatment in Cambodia. Transactions of the Royal Society of Trans R Soc Trop Med Hyg 105: 694-703.

23. Choun K, Thai S, Pe R, Lorent N, Lynen L, et al. (2013) Incidence and risk factors for tuberculosis in HIV-infected patients while on antiretroviral treatment in Cambodia. Trans R Soc Trop Med Hyg 107: 235-242.

24. Oyella J, Meya D, Bajunirwe F, Kamya MR (2012) Prevalence and factors associated with cryptococcal antigenemia among severely immunosuppressed HIV-infected adults in Uganda: a cross-sectional study. J Int AIDS Soc 15: 15.

25. Meyer AC, Kendi CK, Penner JA, Odhiambo N, Otieno B, et al. (2013) The impact of routine cryptococcal antigen screening on survival among HIVinfected individuals with advanced immunosuppression in Kenya. Trop Med Int Health 18: 495-503.

26. Milefchik E, Leal MA, Haubrich R, Bozzette SA, Tilles JG, et al. (2008) Fluconazole alone or combined with flucytosine for the treatment of AIDSassociated cryptococcal meningitis. Med Mycol 46: 393-395.

27. Longley N, Harrison TS, Jarvis JN (2013) Cryptococcal immune reconstitution inflammatory syndrome. Curr Opin Infect Dis 26: 26-34.

28. Pappas PG, Chetchotisakd P, Larsen RA, Manosuthi W, Morris MI, et al (2009) A phase II randomized trial of amphotericin B alone or combined with fluconazole in the treatment of HIV-associated cryptococcal meningitis. Clin Infect Dis 48: $1775-1783$. 